\title{
Zum Spannungsfeld zwischen Empathie und sozialer Verantwortung im medizinischen Alltag
}

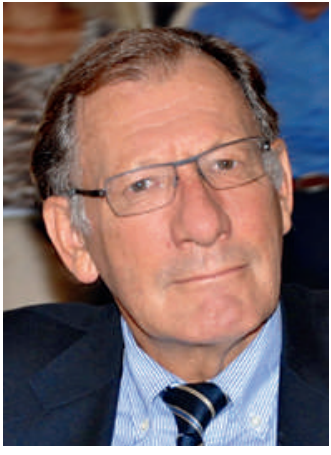

Jean Martin
Ein Chefarzt stellt mir im Kontext seiner Ausbildungsund Führungsverantwortlichkeiten die Frage: «Wie sollen wir mit Kolleginnen und Kollegen, die soziomedizinische Situationen rein empathisch einschätzen, umgehen - wobei der Empathie natürlich grosse Bedeutung zukommt, aber kann sie alleiniges Bewertungskriterium sein?» Er spielt dabei auf die Problematik «zu freundlicher», zu gefälliger Arztzeugnisse an. Er war im Zusammenhang mit Personen angesprochen worden, die Sozialhilfe empfangen und Eingliederungsmassnahmen erhalten sollen, sich aber durch solche Arztzeugnisse entschuldigen lassen und damit potentiell ihre Chancen auf Reintegration in ein aktives Arbeitsleben mindern. Der Kollege engagiert sich sozial und gesellschaftlich und glaubt (wie ich) an einen liberal-sozialen Staat, in dem der Bürger weitreichende Rechte geniesst und - wenn notwendig - Hilfe erhält, aber auch im Rahmen seiner jeweiligen Kompetenzen und Möglichkeiten Verantwortung zu übernehmen hat. Vor diesem Hintergrund sollten die Ressourcen, die das Volk den Krankenkassen und der öffentlichen Hand anvertraut, optimal genutzt werden. Im weiteren Sinne handelt es sich hierbei um eine Frage der Gerechtigkeit.

Wie ist in diesem Zusammenhang bei Ärzten, auch Ärzten in Ausbildung, und anderen Beteiligten, mit denen interagiert wird, vorzugehen? Natürlich ist es wahr, dass der Arzt zunächst einmal seinem Patienten zu dienen hat: «Salus aegroti suprema lex». Aus Sicht der öffentlichen Gesundheit gilt jedoch an der Schnittstelle zwischen Gesundheitsbetreuung und Gesellschaft auch der Satz: «Salus publica suprema lex». Eindeutig lässt sich seit dem Entstehen der allgemeinen Sozialversicherungen (darunter auch die KVG), nicht mehr alles «im Dienste des Patienten» regeln, ohne dabei ausser Acht zu lassen, dass eine maximalistische Praxis auch - da nicht indiziert - zum Nachteil oder sogar Schaden von Personen gereichen kann, deren Bedürfnisse behandelt werden (müssen).

Wie also soll für bestimmte Nebeneffekte von zu gefällig ausgestellten Unfähigkeitsbescheinigungen (in Bezug auf die Arbeit, die Teilnahme an Schulungen usw.) sensibilisiert werden? Es gilt, die Beteiligten des Gesundheitswesens schon im Studium ausdrücklich $\mathrm{zu}$ informieren, mit ihnen darüber zu sprechen, dass Ressourcen immer begrenzt sind, dass «there is no such thing as a free lunch», dass jeder - also auch unser Patient - in ein soziales System eingebettet ist, das zwar nicht vollkommen ist, aber dennoch Vorteile bietet und dass dieses System nicht aus dem Gleichgewicht gebracht werden darf.
Was für die Grundausbildung gilt, gilt auch für die Weiter- und Fortbildung: Kolloquien, Qualitäts- und Diskussionszirkel, Super-/Intervision. Auch interdisziplinär müsste es sich mit Pflegepersonen, Sozialarbeitern und Psychologen bewerkstelligen lassen.

In der Praxis sollte man daran denken, dass jede Organisation, die Menschen beschäftigt (oder, wie im obigen Beispiel, soziale oder bildungsspezifische Leistungen anbietet), die Möglichkeit hat, einen Vertrauensarzt zu benennen, der natürlich völlig unabhängig arbeiten muss. Dieser Vertrauensarzt ist dann befugt, den Kollegen, der das Arztzeugnis ausgestellt hat, um entsprechende und ausreichende Erläuterungen zu bitten, ohne dabei medizinische Details offenzulegen, um dem Betroffenen dann sein Fazit in Bezug auf die Richtigkeit oder - gegebenenfalls - unpräzise oder unbefriedigende Punkte mitzuteilen. Der behandelnde Arzt muss - um dem Vertrauensarzt antworten zu können - vom Patienten von der ärztlichen Schweigepflicht entbunden werden, dieser aber muss sich bewusst sein, dass er Schwierigketien bekommen könnte, wenn der Arzt dem Vertrauenskollegen Fragen nicht beantwortet. In schwerwiegenden Fällen besteht die Möglichkeit, die Situation vor eine Standeskommission oder die kantonale Gesundheitsbehörde zu bringen.

In der Medizin gilt es täglich, sensible Orientierungen oder Entscheidungen zu treffen. Der Arzt soll nicht die Rolle des «Gendarmen» übernehmen, der Missbrauch kontrolliert. Aber es ist zu wünschen, dass Missbrauch nicht oft vorkommt und wir unseren Beitrag leisten können, um ihn zu verhindern. Die Erfahrung zeigt, dass ein Teil unseres Verhaltens immer strittig ist. Wichtig ist, dass sich dieser Teil (sehr) in Grenzen hält.

Es gibt keine Allheilmittel (Leserempfehlungen zu diesem Thema sind willkommen). Ohne Zweifel ist der Arzt berufen, bei Bedarf als Anwalt seines Patienten aufzutreten. Dennoch wird von allen Beteiligten im Gesundheitssystem erwartet, dass sie nach objektiven Kriterien vorgehen (soweit möglich) und sich dabei an wissenschaftliche, ethische und gegebenenfalls juristische Vorgaben halten. Daher gilt es, den Dialog zu fördern, pädagogische Schritte einzuleiten und Überzeugungsarbeit zu leisten. Nicht gleich strafen, aber auch nicht nachlässig handeln.

Last but not least: Ist das «Mitleiden mit dem Patienten» in seiner schwierigen Situation immer sinnvoll, und wie sollte sich dieses Mitleid äussern? Das ist jedoch ein anderes, sehr komplexes Thema ...

Jean Martin, Mitglied der Redaktion 